\title{
Article \\ Dynamic Simulation and Parameter Analysis of Contact Mechanics for Mimicking Geckos' Foot Setae Array
}

\author{
Qing Lin ${ }^{1, *(\mathbb{C})}$, Chunbo $\mathrm{Wu}^{2}{ }^{2} * \mathbb{D}$, Shuai Yue ${ }^{2}$, Zhonghui Jiang ${ }^{3}$, Zhonghua $\mathrm{Du}^{2}$ and Mengsheng $\mathrm{Li}^{2}$ \\ 1 Aerospace System Engineering Shanghai, Shanghai 201109, China \\ 2 School of Mechanical Engineering, Nanjing University of Science and Technology, Nanjing 210094, China; \\ yueshuai@njust.edu.cn (S.Y.); duzhonghua@aliyun.com (Z.D.); lms@njust.edu.cn (M.L.) \\ 3 Xi'an North Qinghua Electrical Co., Ltd., Xi'an 710025, China; shiwenhui@njust.edu.cn \\ * Correspondence: linqing@nuaa.edu.cn (Q.L.); wcb@njust.edu.cn (C.W.)
}

Citation: Lin, Q.; Wu, C.; Yue, S.; Jiang, Z.; Du, Z.; Li, M. Dynamic Simulation and Parameter Analysis of Contact Mechanics for Mimicking Geckos' Foot Setae Array. Crystals 2022, 12, 282. https://doi.org/ $10.3390 /$ cryst12020282

Academic Editors: Yong He, Wenhui Tang, Shuhai Zhang, Yuanfeng Zheng, Chuanting Wang, George Z. Voyiadjis and

Shujun Zhang

Received: 23 January 2022

Accepted: 16 February 2022

Published: 18 February 2022

Publisher's Note: MDPI stays neutral with regard to jurisdictional claims in published maps and institutional affiliations.

Copyright: (c) 2022 by the authors. Licensee MDPI, Basel, Switzerland. This article is an open access article distributed under the terms and conditions of the Creative Commons Attribution (CC BY) license (https:/ / creativecommons.org/licenses/by/ $4.0 /)$.

\begin{abstract}
According to the dynamic characteristics of the adhesion desorption process between gecko-like polyurethane setae and the contact surface, the microcontact principle of an elastic sphere and plane is established based on the Johnson-Kendall-Robert model. On this basis, combined with the cantilever beam model, microscale adhesive contact models in the case of a single and an array of setae are obtained. The contact process is numerically simulated and verified by the adhesion desorption test. After that, the effects of external preload, the elastic modulus of setae material, the surface energy, and the surface roughness on the contact force and depth during the dynamic contact process of setae are studied. The results show that the error between the simulation and test is $15.9 \%$, and the simulation model could reflect the real contact procedure. With the increase in preload, the push-off force of the setae array would grow and remain basically constant after reaching saturation. Increasing the elastic modulus of setae material would reduce the contact depth, but have little effect on the maximum push-off force; with the increase in the surface energy of the contact object, both the push-off force between the objects and the contact depth during desorption would increase. With the increase in wall roughness, the push-off force curve of the setae array becomes smoother, but the maximum push-off force would decrease. By exploring the dynamic mechanical characteristics of the micro angle of setae, the corresponding theoretical basis is provided for the numerical simulation of the adsorption force of macro materials.
\end{abstract}

Keywords: Johnson-Kendall-Robert model; polyurethane setae array; surface energy; modeling; simulation

\section{Introduction}

In recent years, substantial progress has been achieved in gecko-inspired adhesive technology since the discovery of the uniquely layered footpad structure of geckos $[1,2]$. Geckos can climb on almost any surface, or even stand upright or upside down, because their layered structure is composed of inclined villi, and the end of each villus is composed of many thin setae [3]. Researchers have been able to understand the adhesion mechanism of gecko feet and have promoted the development of biomimetic adhesion materials [4-6]. Based on the van der Waals force contact principle, biomimetic adhesive materials may exhibit stable contact performance in complex environments $[7,8]$. The van der Waals force exists in the molecules on the surface of various objects without any requirements regarding the environment, so it is employed by animals such as geckos to adhere to the surfaces of various objects [9].

A lot of research has been carried out on the design and production of gecko-like adhesive materials and structures at home and abroad. In 2000, Autumn et al. [10,11] studied the adhesion mechanism of gecko setae. In the United States, there has been an upsurge in research on biomimetic dry adhesive materials and structures. The adhesion mechanism of gecko setae dominated by the van der Waals force and supplemented by 
the capillary force has been widely accepted. However, the elastic deformation of the setae was rarely involved, leading to the overestimation of the adhesion to the setae. In 2003, Geim et al. proposed a "gecko tape" material, which is fabricated with an array of geometrically shaped flexible plastic posts. The structure has a repeatable attachment function and self-cleaning capability [12]. Persson proposed a simple model to study the effect of surface roughness on the adhesion of gecko foot setae. This model simplifies the tongue depressor at the end of the setae and regards the tongue depressor as an equivalent plane. Assuming that the surface roughness follows a normal distribution, the simulation results show whether the adhesion depends on the magnitude of the surface roughness [13,14]. The literature carries out experimental and theoretical analyses on adhesion to rough surfaces, but does not emphasize the change in the adhesion force of microscopic setae during adhesion. In 2005, the Max Planck Institute for Metal Research in Germany simulated setae found on the foot of a gecko, then optimized and simulated the pulling process of the gecko setae, but lacked research on the adhesion properties of array materials $[15,16]$. Dai Liming et al., of the University of Dayton in the United States, recently used chemical vapor deposition to obtain high-density, large aspect ratio carbon nanotube-adhered arrays [17]. The literature did not further elaborate on the adhesion principle of the array material. Guo et al. studied the dry adhesion of VACNTs in different temperature ranges and explained the phenomenon of adhesion with temperature [18]. The microscopic morphology of the material was optimized by Mark Cutkosky et al. at Stanford University to develop a millimeter-scale array with sharp ends. The adhesion strength of the experimentally obtained adhesion array is about $0.24 \mathrm{~N} / \mathrm{cm}^{2}$, with obvious anisotropy, but the connection between the microscopic setae adhesion theory and the macroscopic adhesion has not been explained further $[19,20]$.

Research on the adhesion characteristics of geckos has good references regarding the development of bionic adhesion materials. Therefore, we could explore the principle of the microstructure of gecko feet to study the adhesion characteristic of polyurethane setae array materials. The traditional Hertz contact theory is mainly used for the contact between two elastomers. When the research scale is further reduced and the surface energy is introduced, the Hertz contact theory cannot be explained. Based on the Hertz theory, the Johnson-Kendall-Robert (JKR) contact theoretical model is established by introducing surface adhesion. The dynamic mechanical properties of setae in microcontact are further studied by integrating the JKR contact theory model with the cantilever beam model. Through the established mathematical model, the influence mechanism of roughness, surface energy, and elastic modulus on the microcontact dynamic characteristics of polyurethane, the setae array is analyzed.

The structure of this paper is as follows: Section 1 summarizes the relevant research and the main contributions of this work. In Section 2, the microscale adhesion theory and cantilever beam theory are studied and integrated to form the setae quasistatic contact theory. Descriptions of experiments, simulations, and the model analysis are included in Section 3. Section 4 concludes the whole research.

\section{Micro Contact Theory of Setae}

\subsection{Microscale Adhesive Contact Theory}

In the macroscopic theory, when objects are in contact with each other, the elastic force is much larger than the surface force of the objects. Thus, the surface force is often ignored. However, when the characteristic scale of the research object is reduced to a certain range, there can be many phenomena that cannot be explained by the traditional macroscopic contact theory. The reason is that the role of the surface force and surface energy between two objects is the key factor in determining the adhesion, contact, and deformation behavior of solid surfaces. In areas where surface forces are dominated, traditional continuum mechanics methods are no longer applicable. The concept of surface force was introduced in classical mechanics; thus, forming the theory of the microscale adhesive contact. 
In the Hertz contact model, due to the lack of an adhesion force, when the applied external load is $P_{h}$, a contact circular surface with a radius $a_{h}$ would be generated, and its contact depth $\delta_{h}$ and pressure distribution $P_{h}(r)$ in the contact area could be given by the following formula:

$$
\begin{gathered}
a_{h}^{3}=\frac{3 R P_{h}}{4 E^{*}} \\
a_{h}^{3}=\frac{3 R P_{h}}{4 E^{*}} \\
p_{h}(r)=\frac{3 P_{h}}{2 \pi a_{h}^{2}}\left(1-\frac{r^{2}}{a_{h}^{2}}\right)^{1 / 2}
\end{gathered}
$$

where $E^{*}$ is the equivalent elastic modulus, $E^{*}=\left(\frac{1-v_{1}^{2}}{E_{1}}+\frac{1-v_{2}^{2}}{E_{2}}\right)^{-1}$ and $R$ are the equivalent radius $\frac{1}{R}=\frac{1}{R_{1}}+\frac{1}{R_{2}}, R_{1} R_{2}$ are the radii of two contact spheres, respectively, and $r$ is the distance between the contact point and the center of the contact area.

Based on the Hertz contact model, the adsorption force $P_{a}$ between the contact surfaces is introduced, and its pressure distribution $P_{h}(r)$ in the contact area could be expressed by the following formula:

$$
P_{a}(r)=\frac{P_{a}}{2 \pi a^{2}}\left(1-\frac{r^{2}}{a_{h}^{2}}\right)^{-1 / 2}
$$

The adsorption term $P_{a}$ has a crack propagation singularity at the contact boundary, and the corresponding pressure increase coefficient is $K_{I}=\frac{P_{a}}{2 a \sqrt{\pi a}}$. Using the Irwin formula, the relationship between the adsorption energy and the adsorption energy could be obtained as:

$$
G=\frac{K_{I}^{2}}{2 E^{*}}=w
$$

Among them, $G$ is the elastic energy release rate, $w$ is the adhesion energy, and $w=2 \sqrt{\gamma_{1} \gamma_{2}}, \gamma_{1}$, and $\gamma_{2}$ are the surface free energy of the two objects in contact with each other, respectively. The expression of the adsorption term could be obtained from the above formula:

$$
P_{a}=\sqrt{8 \pi w E^{*} a^{3}}
$$

The final JKR theoretical contact pressure distribution can be obtained:

$$
P_{J K R}=P_{h}-P_{a}=\frac{4 E^{*} a^{3}}{3 R}-\sqrt{8 \pi w E^{*} a^{3}}
$$

The expression of the contact circle radius $a_{J K R}$ is:

$$
a_{J K R}^{3}=\frac{3 R}{4 E^{*}}\left(P+3 \pi w R+\sqrt{6 \pi w R P+(3 \pi w R)^{2}}\right)
$$

The corresponding normal displacement is $\delta_{J K R}$ and could be written as:

$$
\delta_{J K R}=\delta_{h}+\delta_{a}
$$

where $\delta_{h}$ is the Hertz compression depth, $\delta_{a}$ is the adsorption compression depth, and $\delta_{a}=\frac{P_{a}}{2 \pi E^{*}}=\frac{\sqrt{8 \pi E^{*} w a^{3}}}{2 \pi E^{*}}$; finally, the normal displacement could be written as:

$$
\delta_{J K R}=\frac{a_{J K R}^{2}}{R}-\sqrt{\frac{2 \pi w a_{J K R}}{E^{*}}}
$$


To facilitate the analysis of the essence of the JKR contact model, the obtained dimensionless external load $P^{*}$, contact circle radius $a^{*}$, and contact depth $\delta^{*}$ are:

$$
\begin{gathered}
P^{*}=\frac{P_{J K R}}{\pi R w} \\
a^{*}=a_{J K R}\left(\frac{4 E^{*}}{3 \pi R^{2} w}\right)^{1 / 3} \\
\delta^{*}=\delta_{J K R}\left(\frac{9 \pi^{2} R w^{2}}{16 E^{* 2}}\right)^{-1 / 3}
\end{gathered}
$$

Then, according to the formula (7), the expression of dimensionless load $P^{*}$ could be obtained as:

$$
P^{*}=P_{h}^{*}-\left(6 P_{h}^{*}\right)^{1 / 2}=a^{* 3}-\left(6 a^{* 3}\right)^{1 / 2}
$$

Based on Equation (10), so that the contact depth $\delta^{*}$ :

$$
\delta^{*}=a^{* 2}-2\left(2 a^{*} / 3\right)^{1 / 2}
$$

When the external load is 0 , the contact radius generated by the van der Waals force is $a_{0}=\left({ }^{6 \pi R^{2} w} / K^{1 / 3}\right)$, and the following could be seen from Equation (8): the maximum adhesion force: $F_{a d}=-1.5 \pi R w$; the separation radius: $a_{s}=0.63 a_{0}$; the normal displacement during separation: $\delta_{s}=-0.21 a_{0}^{2} R^{-1}$.

\subsection{Cantilever Beam Theory}

Since the $J K R$ theory could only describe the micro-scale adhesion properties and cannot characterize the elastic deformation of the setae rods, it may lead to the overestimation of the adhesion strength. When analyzing the microscopic contact of setae, a single seta rod could be regarded as a large flexible beam, and the end could be simplified as a viscous elastic sphere. This simplified model could deal with issues related to setae contact.

By applying vertical pressure on the setae, they can gradually achieve close contact with the surface. The setae would then be in the adsorption state. After that, a push-off force could be applied on the setae to separate them from the contact surface. Based on Figure 1, the mechanical analysis of a single polyurethane seta was carried out.

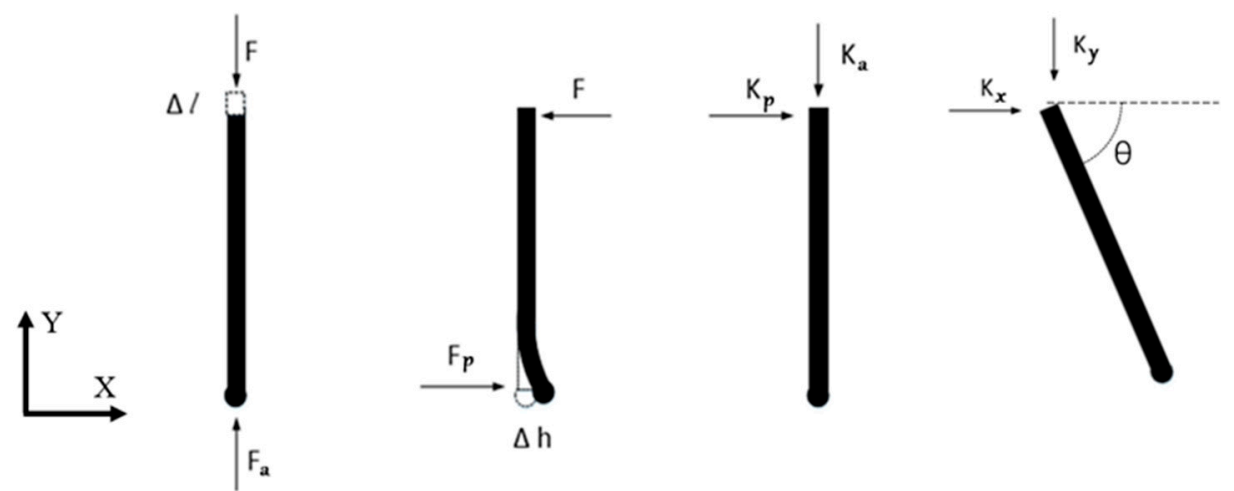

Figure 1. Schematic diagram of the stiffness of a single seta. 
Neglecting the instability, when the setae were compressed in their axial direction, the ends of the setae were also subjected to a reaction force in the axial direction. According to the deformation theory of large flexible beams in the mechanics of materials, the deformation of the seta in their axial direction was:

$$
\Delta l=\frac{F l}{\pi r^{2} E_{1}}
$$

where $l$ is the length of the setae rod, $E_{1}$ is the elastic modulus of the setae material, $r$ is the diameter of the setae rod, and $F$ is the axial load on the setae. The above Equation (16) could be transformed into a function $F=K_{a} \Delta l$, where $K_{a}$ is the stiffness of the setae along the axial direction, with the expression:

$$
K_{a}=\frac{\pi r^{2} E_{1}}{l}
$$

when the end of the setae is subjected to an external force along its radial direction, the bending deformation of the material would cause a relative displacement $\Delta h$ in the radial direction. Therefore:

$$
\Delta h=\frac{F l^{3}}{3 E_{1} I}
$$

The above formula could be transformed into a function $F=K_{p} \Delta h$, where $K_{p}$ is the stiffness of the setae along the radial direction, and its expression is:

$$
K_{p}=\frac{F}{\Delta h}=\frac{3 \pi r^{4} E}{4 l^{3}}
$$

The longitudinal stiffness $K_{y}$ of the setae at the inclination angle $\theta$ could be expressed as:

$$
K_{y}=\frac{F_{y}}{\delta_{y}}=\frac{K_{P} \cdot K_{a}}{K_{P} \sin ^{2} \theta+K_{a} \cos ^{2} \theta}
$$

In the same way, the expression of the lateral stiffness $K_{X}$ of the setae could be expressed as:

$$
K_{x}=\frac{F_{x}}{\delta_{x}}=\frac{K_{P} \cdot K_{a}}{K_{P} \cos ^{2} \theta+K_{a} \sin ^{2} \theta}
$$

To facilitate the study of the plastic deformation of the seta rod, the axial stiffness and the radial stiffness of the inclined seta rod were converted into the longitudinal stiffness $K_{y}$ and the transverse stiffness $K_{X}$. The comprehensive deformation of the seta rod could be decomposed into the $X$-direction deformation and the $Y$-direction deformation according to the coordinate system.

\subsection{Quasi-Static Contact Theory}

When the setae were pressed in or pulled out vertically at a low speed, the contact state of a single polyurethane seta with the surface could be approximately regarded as the quasistatic contact. As shown in Figure 2, the distance between the base of the setae and the contact surface was $Z$, the length of the setae rod was $L$, and the radius of the end ball was $R$. The seta was displaced as $y$ in the $Y$ direction. The initial distance of the setae from the contact plane was $h_{0}$. The distance between the end of the seta and the contact surface during the movement was $h$.

$$
h=Z-L \sin \theta-R-y
$$




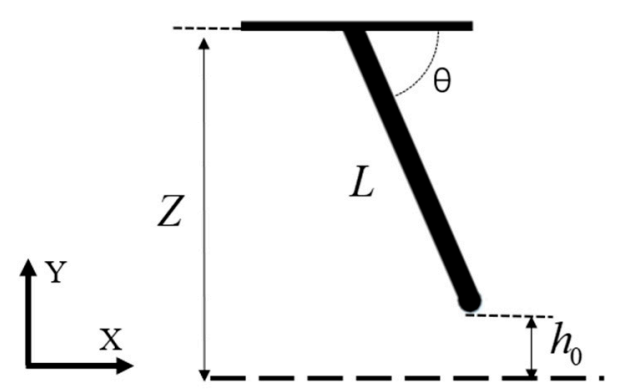

Figure 2. Schematic diagram of single polyurethane setae and surface.

The setae contact state was judged by analyzing the size of $h$. Since the interaction force of the setae in the process of contact and desorption was different, it was necessary to analyze the mechanical state of the pressing and desorption of the polyurethane setae.

\subsubsection{Quasistatic Indentation Force Analysis of Setae}

The polyurethane setae were pressed vertically in the $Y$ direction. Due to the van der Waals forces acting at a small distance, the polyurethane setae were just in contact with the surface without deformation at that time $h=0$. The interaction force between the polyurethane setae and the surface was 0 at this time. When $h<0$, the polyurethane setae were squeezed, and the interaction force between them was expressed as a repulsive force $F>0$.

The displacement of the base of the setae was $y$, and the contact depth between the ball at the end of the setae was $y_{1}$. When the rod of the setae was compressed, its elastic deformation was $y_{2}$. As shown in Figure $3 a$, the relationship between their displacements could be obtained:

$$
y=y_{1}+y_{2}
$$

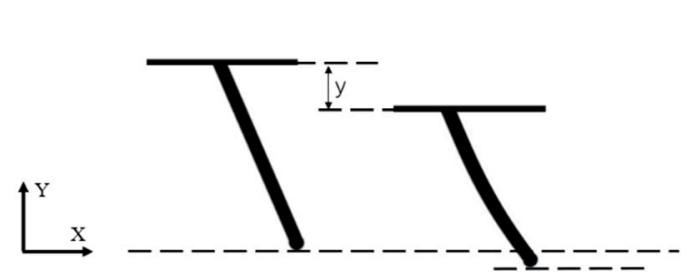

(a)

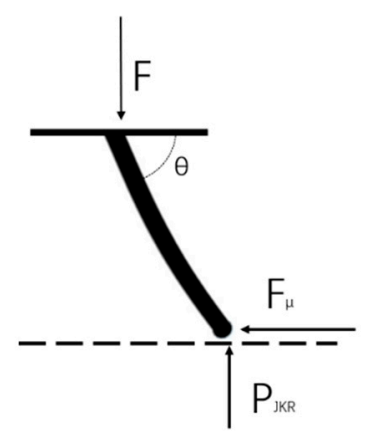

(b)

Figure 3. Static relationship between seta and contact surface;(a) variation of a single seta press-in displacement;(b) force analysis of a single seta in contact with the surface.

As the setae moved down, the displacement $y_{1}$ for the end ball was the $J K R$ theoretical depth of contact:

$$
y_{1}=\delta_{J K R}
$$

Based on the force balance theory, as shown in Figure 3b, we could obtain:

$$
F=P_{J K R}=y_{2} \times K_{y}
$$

By combining Equations. (7), (10), (20), (23), and (25), the relationship between the contact force and the displacement of the setae in the $Y$ direction could be obtained. 
During the pressing process, the seta would produce a lateral deformation $x$, which could be approximated according to the deformation coordination relationship:

$$
x=\left(y-y_{1}\right) \times \tan \theta
$$

The coefficient of friction between the setae and the surface was $\mu$, and the setae experienced a leftward frictional force $F_{\mu}$ during the press-in process:

$$
F_{\mu}=\mu \times F
$$

If the force generated by the lateral deformation of the setae was less than the friction force, that is $x K_{x} \leq \mu F$, then the setae would not produce a lateral displacement. Additionally, the friction force value was equal to the static friction. If the force generated by the lateral deformation of the setae was greater than the friction force, that is $x K_{x}>\mu F$, then the setae would produce a lateral displacement. Additionally, the friction force value at this time was equal to the size of the kinetic friction force.

\subsubsection{Quasistatic Push-off Force Analysis of Setae}

The polyurethane setae were perpendicular to the surface in the $Y$ direction. When $h<0$, the polyurethane setae were squeezed, and the interaction force between them was expressed as the repulsive force $F>0$. In the $Y$ direction, the contact force between the setae and the surface was the same as that in the pressed state. The friction force was opposite to that in the pressed state in the $X$ direction.

When $\delta_{s} \geq h>0$, the polyurethane setae end beads adhered to the surface, and the interaction force between them was an attractive force $F<0$, as shown in Figure 4 . Equation (25) was also applicable to the solution of the contact force in the desorption state, but the force state was opposite to that in the press-in state.

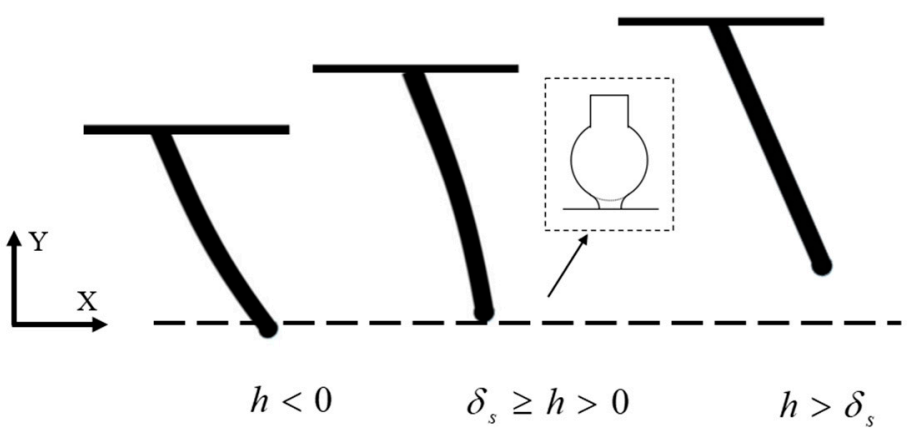

Figure 4. Schematic diagram of desorption of single polyurethane setae.

When $h>\delta_{s}$ the polyurethane setae were separated from the surface, and the interaction force between them was $F=0$.

\section{Results and Discussion}

\subsection{JKR Model Analysis}

By solving the normalized $J K R$ model of the setae contact, the relationship between the contact circle radius, contact force, and contact depth was obtained. Through the analysis of the curve characteristics, the dynamic mechanical relationship between the two objects in the mutual adsorption and desorption process was obtained.

The relationship between the force and the radius when the setae were in contact with the surface is shown in Figure 5a. It could be seen from the figure that the maximum adsorption force was at point $C$. The corresponding adsorption force was $-1.5 \mu \mathrm{N}$ at this point, and the radius of the contact circle was $1.24 \mu \mathrm{m}$. This point was the critical point where the ball end of the setae and the contact surface were separated from each other. 
When the ball at the end of the setae was 0 from the surface, the adsorption force changed from point $\mathrm{O}(0,0)$ to point $\mathrm{A}(0,-1.35 \mu \mathrm{N})$ in Figure $5 \mathrm{~b}$. The increase in the contact depth led to a growth in the adsorption force, which changed in the $\mathrm{AB}$ direction from point $\mathrm{A}$. When the ball surface and the contact surface were separated from each other, the force would gradually decrease along with BA. The positive pressure gradually became the adsorption force, and then changed from point $\mathrm{A}$ to point $\mathrm{D}(-0.8,-0.75)$. When it was further detached from point $\mathrm{D}$, the adsorption force would change suddenly from point $\mathrm{D}(-0.8,-0.75)$ to point $\mathrm{E}(-0.8,0)$, which also meant that the polyurethane setae were completely detached from the contact surface. Point $\mathrm{D}$ was the critical point, where the polyurethane setae and the contact surface were separated from each other.

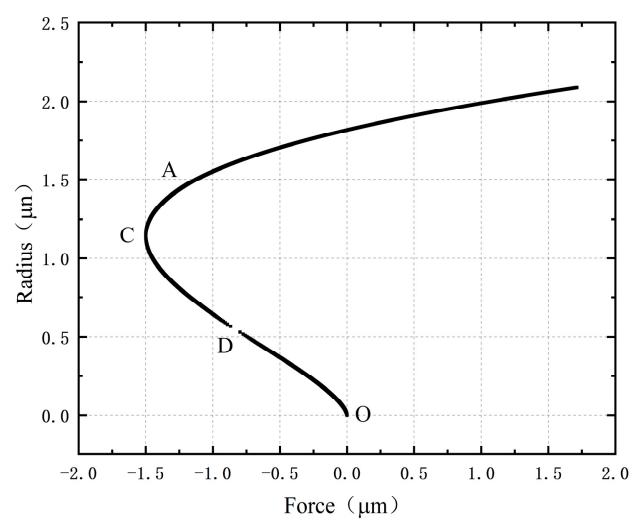

(a)

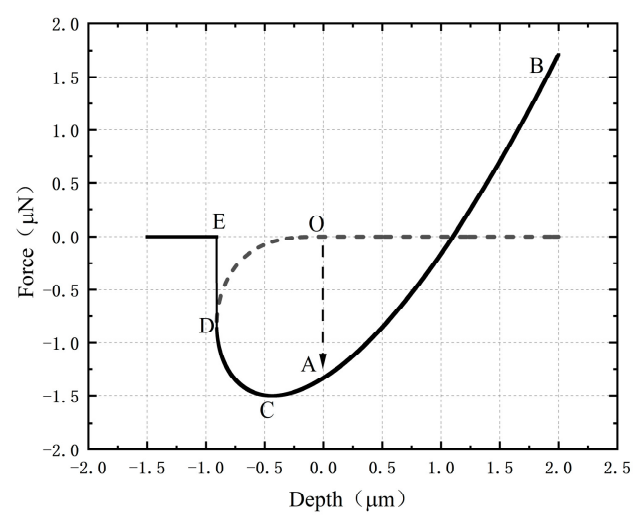

(b)

Figure 5. The dynamic mechanical relationship between the sphere and the contact surface: (a) the relationship between the radius and the force; $(\mathbf{b})$ the relationship between the contact force and the contact depth.

\subsubsection{Influence by Elastic Modulus}

Based on the above theory, the effect of the material's elastic modulus and surface energy on the interaction force model was further analyzed. The dynamic mechanical simulation analysis was carried out by selecting real polyurethane seta material parameters. The sphere and the plane were selected to be in contact with each other, the radius of the sphere at the end of the polyurethane setae was $4 \mu \mathrm{m}$, the elastic modulus was $1.413 \mathrm{Mpa}$, the Poisson's ratio was $0.3 \mathrm{~mJ} / \mathrm{m}^{2}$, and the adhesion energy was $40 \mathrm{~mJ} / \mathrm{m}^{2}$. The elastic modulus of the contact plane was $55 \mathrm{Gpa}$, the Poisson's ratio was 0.25 , and the adhesion energy was $170 \mathrm{~mJ} / \mathrm{m}^{2}$.

Taking the elastic modulus as $0.1 \mathrm{Mpa}, 1 \mathrm{Mpa}$, and $10 \mathrm{Mpa}$ for the dynamic simulation, we could obtain the relationship curve between the circle radius and the external load, and the external load and the depth. It could be seen from Figure 6a that with the increase in the elastic modulus, the contact circle radius under the same contact force decreased significantly from $3.69 \mu \mathrm{m}$ at $0.1 \mathrm{Mpa}$ to $0.74 \mu \mathrm{m}$ at $10 \mathrm{Mpa}$. An increase in the elastic modulus resulted in less deformation under the same contact force. Under the same contact force, the deformation became smaller as the elastic modulus increased. It was also found that the maximum push-off force of spheres with different elastic moduli experienced little change during the separation process. 


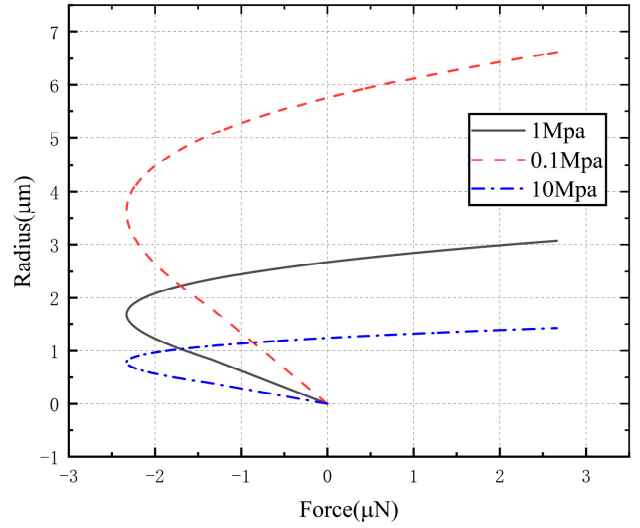

(a)

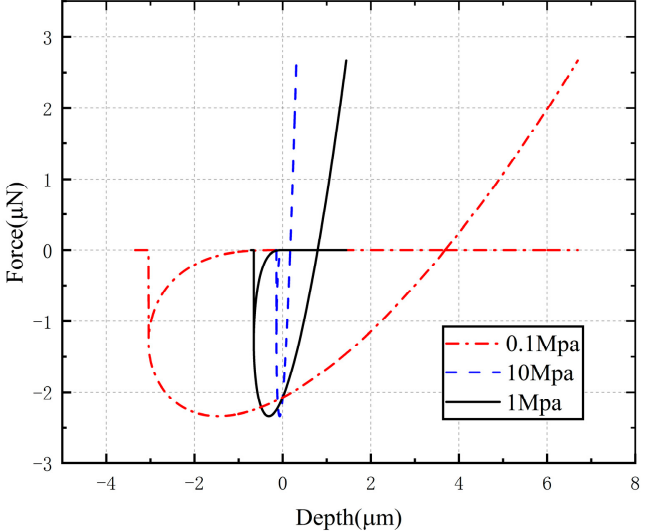

(b)

Figure 6. Dynamic analysis results under different elastic moduli of $0.1 \mathrm{Mpa}, 1 \mathrm{Mpa}$, and $10 \mathrm{Mpa}$ : (a) the relationship between the contact radius and the contact force under different elastic moduli; (b) the relationship between the contact force and the contact depth under different elastic moduli.

\subsubsection{Influence by Material Surface Energy}

Taking the sphere adhesion energy as $4 \mathrm{~mJ} / \mathrm{m}^{2}, 40 \mathrm{~mJ} / \mathrm{m}^{2}$, and $100 \mathrm{~mJ} / \mathrm{m}^{2}$, the dynamic simulation was carried out. The relationship between the radius of the circle and the load, and the load and the depth were obtained. It could be seen from Figure $7 \mathrm{a}$ that with the increase in the surface adhesion energy, the maximum adsorption force when the setae were desorbed increased significantly. The maximum adsorption force increased from $0.71 \mu \mathrm{N}$ at $4 \mathrm{~mJ} / \mathrm{m}^{2}$ to $3.55 \mu \mathrm{N}$ at $100 \mathrm{~mJ} / \mathrm{m}^{2}$. With the increase in the surface adhesion energy of the contacting object, the corresponding contact depth also increased gradually during desorption, from $0.23 \mu \mathrm{m}$ at $4 \mathrm{~mJ} / \mathrm{m}^{2}$ to $0.71 \mu \mathrm{m}$ at $100 \mathrm{~mJ} / \mathrm{m}^{2}$.

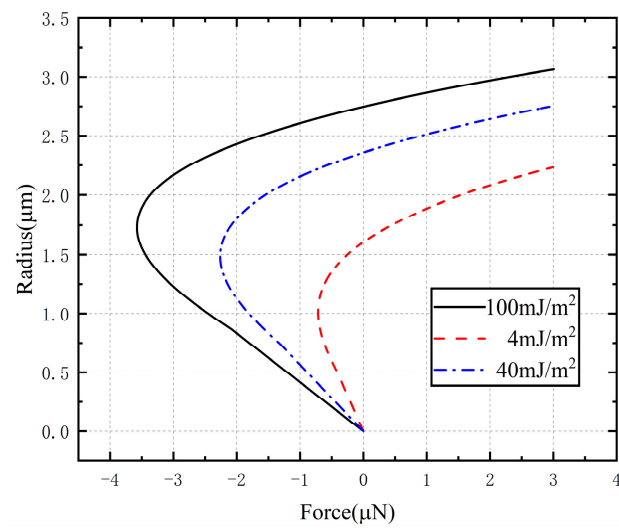

(a)

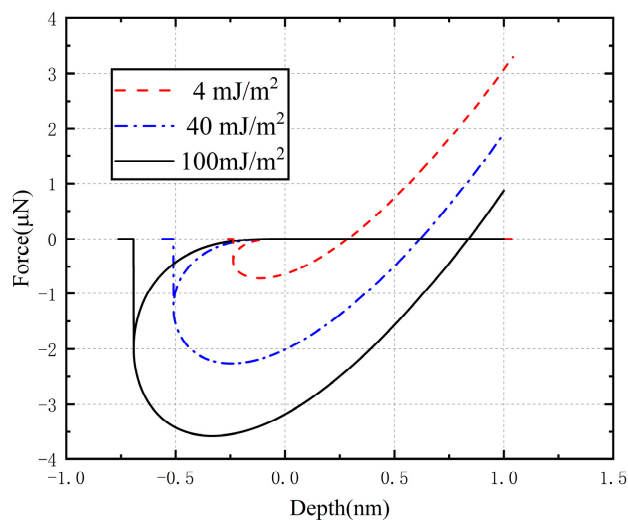

(b)

Figure 7. Dynamic analysis results under the conditions of different surface energies of $4 \mathrm{~mJ} / \mathrm{m}^{2}$, $40 \mathrm{~mJ} / \mathrm{m}^{2}$, and $100 \mathrm{~mJ} / \mathrm{m}^{2}$ : (a) the relationship between the contact radius and force under different surface energies; (b) the relationship between the contact force and depth under different surface energies.

To sum up, the contact depth would increase with the decrease in the elastic modulus under the same contact load, but had little effect on the maximum push-off force. The depth of the contact and maximum push-off force would increase as the surface energy of the contacting object increased. Therefore, the main factor affecting the setae push-off force was the surface energy of the material. When the material for creating the setae was determined, no matter how the morphology and size of the setae changed, the value of the push-off force was constant. 


\subsection{Simulation of the Single Seta}

The interaction force between a single seta and the surface adhesion or detachment had different forms, so the micro-adhesion state of the setae needed to be considered and analyzed separately. When $\delta>0$, the seta would be in contact with the surface and elastically deform. When $\delta<0$, the polyurethane seta would undergo a plastic deformation due to the effect of adhesion and remained in contact with the surface. Until the elastic force overcame the adhesion $\delta>\delta_{0}$, the setae detached from the surface.

The dynamic desorption process of a single polyurethane seta was simulated and analyzed, and the parameters were selected as shown in Table 1 . The dynamic desorption process of setae was solved by Matrix Laboratory (MATLAB) programming.

Table 1. Selection of polyurethane setae parameters.

\begin{tabular}{ccccccc}
\hline Material Name & Slope & Setae Diameter & Length & Poisson's Ratio & Elastic Modulus & Adhesive Energy \\
\hline Polyurethane & $30^{\circ}$ & $3 \mu \mathrm{m}$ & $20 \mu \mathrm{m}$ & 0.3 & $1.413 \mathrm{Mpa}$ & $40 \mathrm{~mJ} / \mathrm{m}^{2}$ \\
\hline
\end{tabular}

The relationship curve between the contact force and the contact depth was obtained through a simulation, as shown in Figure 8. The setae were slowly pulled out at a certain speed, and the contact force decreased with the contact depth and changes toward BA. When the setae were pulled apart to $-7.2 \mu \mathrm{m}$, the contact was suddenly disconnected. From point $\mathrm{D}$ to point $\mathrm{E}$, the adsorption force suddenly changed from $4.1 \mu \mathrm{N}$ to $0 \mathrm{~N}$. The EF segment setae were separated from the contact surface, and there was no interaction force detected.

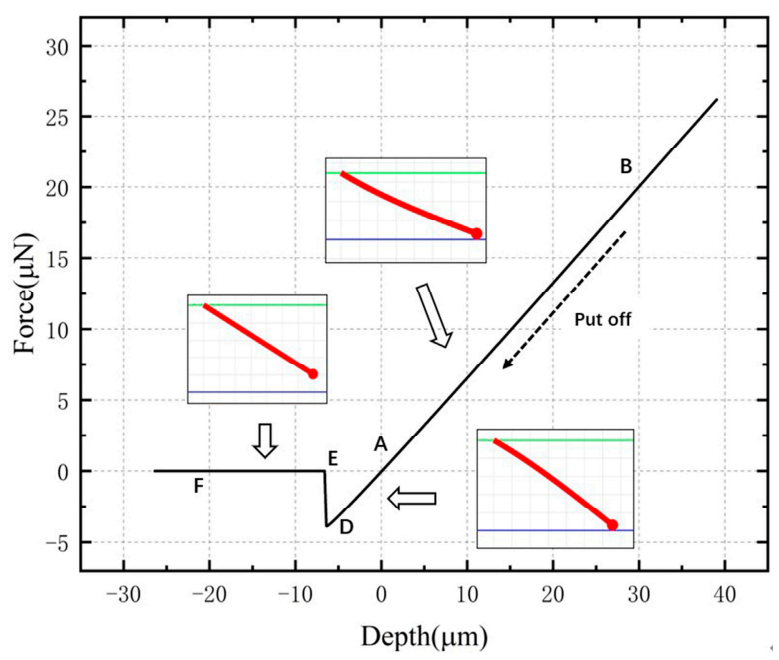

Figure 8. Simulation curve of contact force and contact depth for setae array.

\subsection{Setae Array Simulation and Experiment \\ 3.3.1. Rough Surface Model}

An important assumption of the classical contact theory is that the contact surface is geometrically smooth, but the real surface in engineering is rough. Therefore, it was necessary to establish a roughness model that could approximate the actual surface and could be easily calculated. As shown in Figure 9, assuming that the base surface of the setae was parallel to the reference plane of the contact surface, the spacing was $Z$, the length of each seta was $L$, the setae were parallel to each other, and the angle between the setae and the base surface was $\theta$. 


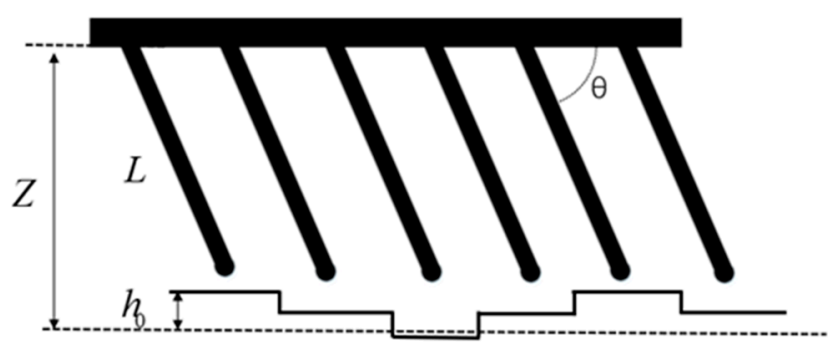

Figure 9. Roughness model.

It was assumed that the peak height distribution of the contact surface conformed to a normal distribution, and the reference surface of the rough surface was a plane. The distance was $h$ between the contact surface profile and the reference plane, and the height satisfied the following Gaussian distribution:

$$
\mathrm{g}(h)=\frac{1}{\sqrt{2 \pi \sigma}} e^{-\frac{h^{2}}{2 \sigma^{2}}}
$$

\subsubsection{Dynamic Simulation and Experimental Verification}

The dynamic adhesion process of polyurethane setae arrays was simulated and analyzed, and the setae were pulled out vertically from the bottom to the top. When the material area was $1 \mathrm{~cm}^{2}$ and the seta spacing was $4 \mu \mathrm{m}$, there was $1 \times 10^{7}$ setae in total, and the inclination angle of the seta was $60^{\circ}$. The elastic modulus was taken as $1.413 \mathrm{Mpa}$, the Poisson's ratio was 0.3 , the surface adhesion energy was $40 \mathrm{~mJ} / \mathrm{m}^{2}$, and the friction coefficient between the end of the setae and the surfaces was 0.2 . The elastic modulus of the contact surface was taken as $55 \mathrm{Gpa}$, the Poisson's ratio was 0.25 , and the adhesion energy was taken as $170 \mathrm{~mJ} / \mathrm{m}^{2}$. The mean square error of the surface roughness was 1 . Using MATLAB programming to solve the dynamic desorption process of setae, the results obtained by dynamic calculation are shown in the following Figure 10.

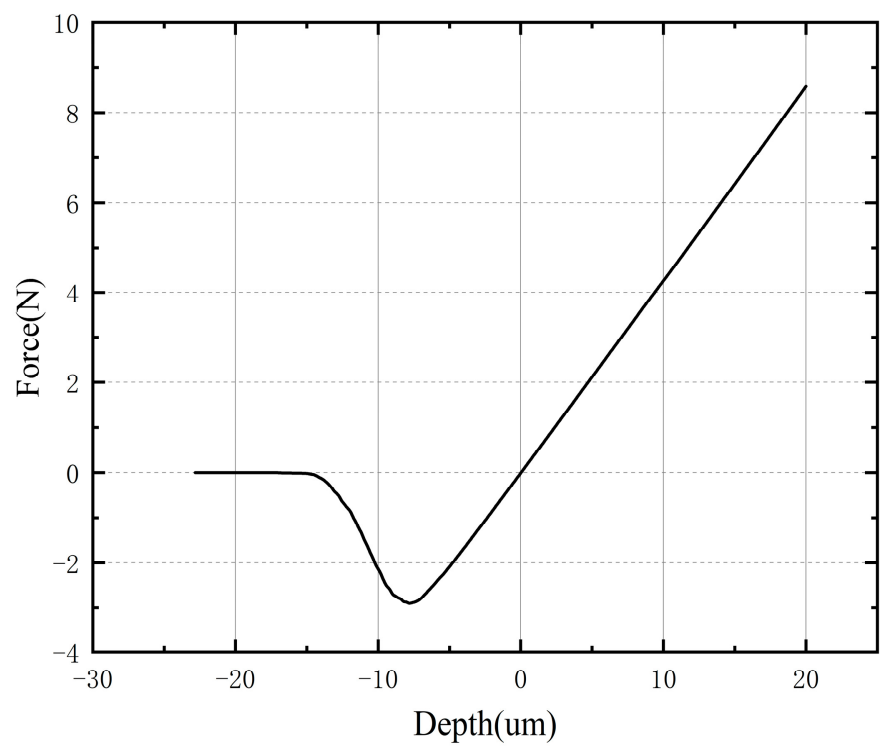

Figure 10. Simulation curve of the contact force with different contact depths.

The relationship between the push-off force and the depth was obtained through a simulation, as shown in Figure 10. With the continuous pulling out of the setae array, the contact force decreased gradually from $8.6 \mathrm{~N}$. When the contact depth was less than $0 \mu \mathrm{m}$, the direction of the contact force changed from pressure to adsorption. The curve had an extreme point as the adsorption force reached a maximum value of $-2.85 \mathrm{~N}$. As the setae 
pulled further apart, the adsorption force gradually decreased to $0 \mathrm{~N}$, and all the setae were separated from the surface.

Figure 11a shows the relationship between the adsorption force and the preload on the setae array when the mean square error of the roughness was 1 . We could see from the simulation curve that the adsorption force of the setae array increased rapidly with the increase in pressure. After the pre-pressure reached $1 \mathrm{~N}$, the adsorption force stabilized at about $2.3 \mathrm{~N}$. The experimental results matched well and the deviation error of the data was $7.81 \%$.

As the preload force increased, the number of setae arrays in contact with the surface increased, so the preload force determined the distribution of adhesion between the array and the surface. Different adhesion distributions would produce different interaction force relationship curves during the detachment process. There was an extreme point of adhesion force in the desorption curve under a certain pre-pressure, as shown in Figure 10. By collecting the maximum desorption force under different pre-pressures, the relationship curve between the pre-pressure and the maximum desorption force was obtained. During dynamic detachment, the maximum adhesion force produced by the setae array increased with an increasing preload, as shown in Figure 11a. The preload was further increased, and the maximum adhesion attraction tended to reach a stable value. Because the area of the material was constant in both experiments and simulations, there was a saturation value for the number of setae contacts. The mean square error of surface roughness was selected as $0.5,1$, and 1.5 , respectively. The experimental and simulation results of setae in contact with surfaces of different roughness are shown in Figure 11b. The root mean square (RMS) was used to describe the dispersion between the experimental data and the simulation data, and the calculation result was $15.9 \%$ [21]. The peak adsorption force increased continuously at different preloads as the root mean square deviation of the surface decreased. The contact of the polyurethane setae array with the rough surface became better as the root mean square deviation decreased, so the smooth surface was more favorable to the adsorption of the setae array.

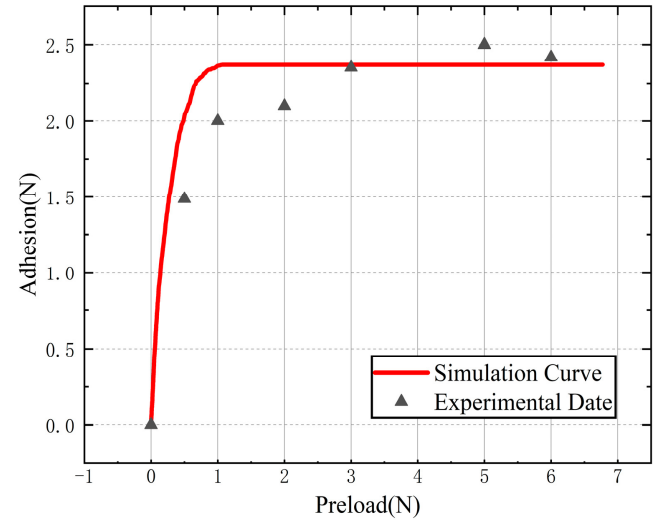

(a)

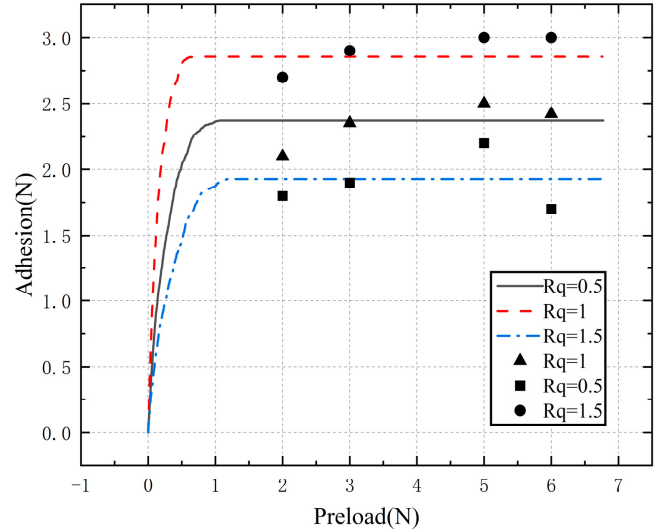

(b)

Figure 11. The relationship curves of the adsorption force between experiment and simulation: (a) results data with different preloads; (b) results data with different surface roughness.

\section{Conclusions}

This paper established a complete contact model for polyurethane setae arrays, which considered the Hertz contact theory, the surface energy theory, the cantilever beam theory, and the rough surface mode comprehensively. When analyzing the contact of a single seta, the setae rod was simplified as a cantilever beam, with its axial and radial stiffnesses converted into longitudinal and transverse stiffnesses. Integrating the single setae quasistatic contact theory and the rough surface model, the setae array contact model was further obtained. After that, a parameter analysis was conducted and verified by the corresponding experimental data. The main conclusions were as follows: 
(1) By comparing the simulation with the experiment, the average discrepancy of the data was $15.9 \%$, which demonstrated the feasibility of the polyurethane setae arrays contact model.

(2) Through the analysis of the JKR model for the ball end of the setae, it was found that reducing the elastic modulus of the object would increase the depth of contact under the same external load. The elastic modulus had little effect on the maximum push-off force. Increasing the surface energy of the contacting object would grow the distance between the objects, the push-off force, and the depth of contact during desorption.

(3) When the polyurethane setae array was in contact with the rough surface, it was found that with the growth of the preload applied to the setae array, the number of setae in contact rose. Its maximum adsorption force would also increase, but it would reach saturation.

(4) Different properties of the contact surface material had different effects on adhesion. The greater the surface energy of the contact surface, the greater the adsorption force generated when the seta arrays were separated. The elastic modulus and Poisson's ratio of the contact surface had little effect on the adsorption force. The roughness of the contact surface had a negative effect on the adsorption force of the seta array, and the smoother the surface, the greater the adsorption force.

Author Contributions: Conceptualization, Q.L. and S.Y.; Software, C.W.; Validation, Z.J., M.L. and Q.L.; Writing-Original Draft Preparation, Q.L. and Z.J.; Writing—Review and Editing, Z.D. and C.W.; Supervision, S.Y. All authors have read and agreed to the published version of the manuscript.

Funding: This work was supported by the National Natural Science Foundation of China (52102436), the Fundamental Research Funds for the Central Universities (30920021109), the Natural Science Foundation of Jiangsu Province (BK20200496), the China Postdoctoral Science Foundation (2020M681615), and the project of Key Laboratory of Impact and Safety Engineering (Ningbo University), the Ministry of Education (CJ202107).

Institutional Review Board Statement: Not applicable.

Informed Consent Statement: Not applicable.

Data Availability Statement: Data are contained within the article. The data presented in this study can be seen in the content above.

Acknowledgments: We wish to express our gratitude to the members of our research team, Shuai Yue, Chunbo $\mathrm{Wu}$, Zhonghui Jiang, Zhonghua Du, and Mengsheng Li.

Conflicts of Interest: The authors declare no conflict of interest.

\section{References}

1. Cadirov, N.; Booth, J.A.; Turner, K.L.; Israelachvili, J.N. Influence of humidity on grip and release adhesion mechanisms for gecko-inspired microfibrillar surfaces. ACS Appl. Mater. Interfaces 2017, 9, 14497-14505. [CrossRef] [PubMed]

2. Wan, Y.; Xia, Z. Self-cleaning and controlled adhesion of gecko feet and their bioinspired micromanipulators. MRS Adv. 2018, 3 , 1641-1646. [CrossRef]

3. Hu, S.; Jiang, H.; Xia, Z.; Gao, X. Friction and adhesion of hierarchical carbon nanotube structures for biomimetic dry adhesives: Multiscale modeling. ACS Appl. Mater. Interfaces 2010, 2, 2570-2578. [CrossRef] [PubMed]

4. Raut, H.K.; Baji, A.; Hariri, H.H.; Parveen, H.; Soh, G.S.; Low, H.Y.; Wood, K.L. Gecko-inspired dry adhesive based on micronanoscale hierarchical arrays for application in climbing devices. ACS Appl. Mater. Interfaces 2018, 10, 1288-1296. [CrossRef] [PubMed]

5. Sitti, M.; Fearing, R.S. Synthetic gecko foot-hair micro/nano-structures for future wall-climbing robots. In Proceedings of the IEEE International Conference on Robotics and Automation (Cat. No. 03CH37422), Taipei, Taiwan, 14-19 September 2003; pp. 1164-1170.

6. Shah, G.J.; Sitti, M. Modeling and design of biomimetic adhesives inspired by gecko foot-hairs. In Proceedings of the IEEE International Conference on Robotics and Biomimetics, Shenyang, China, 22-26 August 2004; pp. 873-878.

7. Peng, M.; Han, X.; Xiao, G.Z.; Chen, X.; Xiong, W.; Yang, K.; Luo, H. Spherical volume elements scheme for calculating van der Waals force between irregular particles and rough surfaces. Chin. J. Phys. 2021, 72, 645-654. [CrossRef]

8. Lee, J.; Bush, B.; Maboudian, R.; Fearing, R. Gecko-Inspired Combined Lamellar and Nanofibrillar Array for Adhesion on Nonplanar Surface. Langmuir 2009, 25, 12449-12453. [CrossRef] [PubMed] 
9. Li, X.; Tao, D.; Lu, H.; Bai, P.; Liu, Z.; Ma, L.; Meng, Y.; Tian, Y. Recent developments in gecko-inspired dry adhesive surfaces from fabrication to application. Surf. Topogr. Metrol. Prop. 2019, 7, 023001. [CrossRef]

10. Autumn, K.; Liang, Y.A.; Hsieh, S.T.; Zesch, W.; Chan, W.P.; Kenny, T.W.; Fearing, R.; Full, R.J. Adhesive force of a single gecko foot-hair. Nature 2000, 405, 681-685. [CrossRef] [PubMed]

11. Autumn, K.; Sitti, M.; Liang, Y.A.; Peattie, A.M.; Hansen, W.R.; Sponberg, S.; Kenny, T.W.; Fearing, R.; Israelachvili, J.N.; Full, R.J. Evidence for van der Waals adhesion in gecko setae. Proc. Natl. Acad. Sci. USA 2002, 99, 12252-12256. [CrossRef] [PubMed]

12. Geim, A.K.; Dubonos, S.V.; Grigorieva, I.V.; Novoselov, K.; Zhukov, A.A.; Shapoval, S.Y. Microfabricated adhesive mimicking gecko foot-hair. Nat. Mater. 2003, 2, 461-463. [CrossRef] [PubMed]

13. Persson, B. On the mechanism of adhesion in biological systems. J. Chem. Phys. 2003, 118, 7614-7621. [CrossRef]

14. Dai, Z.D.; Hui, C.; Stannislav, G. Effect of surface roughness on the adhesive properties of polyurethane. Tribology 2003, 23, 245-249.

15. Gao, H.; Yao, H. Shape insensitive optimal adhesion of nanoscale fibrillar structures. Proc. Natl. Acad. Sci. USA 2004, 101, 7851-7856. [CrossRef] [PubMed]

16. Gao, H.; Xiang, W.; Yao, H.; Gorb, S.; Arzt, E. Mechanics of hierarchical adhesion structures of geckos. Mech. Mater. 2005, 37, 275-285. [CrossRef]

17. Qu, L.; Dai, L.; Stone, M.; Xia, Z.; Wang, Z.L. Carbon nanotube arrays with strong shear binding-on and easy normal lifting-off. Science 2008, 322, 238-242. [CrossRef] [PubMed]

18. Guo, F.; Li, Y.; Meng, G.; Sun, C.; Dai, Z. Adhesion property of vertically aligned carbon nanotubes under high temperature. Trans. Nanjing Univ. Aeronaut. Astronaut. 2018, 35, 41-45.

19. Santos, D.; Spenko, M.; Parness, A.; Kim, S.; Cutkosky, M. Directional adhesion for climbing. theoretical and practical considerations. J. Adhes. Sci. Technol. 2007, 21, 1317-1341. [CrossRef]

20. Chen, R.; Fu, L.; Qiu, Y.; Song, R.; Jin, Y. A gecko-inspired wall-climbing robot based on vibration suction mechanism. Proc. Inst. Mech. Eng. Part C J. Mech. Eng. Sci. 2019, 233, 7132-7143. [CrossRef]

21. Yue, S.; Titurus, B.; Nie, H.; Zhang, M. Liquid spring damper for vertical landing Reusable Launch Vehicle under impact conditions. Mech. Syst. Signal Process. 2019, 121, 579-599. [CrossRef] 